\title{
Quality of Service (QoS): Congestion and Queuing
}

\author{
Mahesh VR ${ }^{1}$, Ankush Reddy $P^{2}$, Charan HM$^{3}$, Anil Umesh ${ }^{4}$ \\ BE, Department of ECE, BIT, Bangalore, India ${ }^{1}$ \\ BE, Department of TCE, Sir MVIT, Bangalore, India ${ }^{2}$ \\ BE, Department of ECE, BIT, Bangalore, India $^{3}$ \\ BE, Department of CSE, SJCE, Mysore, India ${ }^{4}$
}

\begin{abstract}
Quality of Service (QoS) refers to ability of a network to provide improved service to selected network traffic over various underlying technologies including Frame Relay, ATM, Ethernet and 802.1 networks, SONETS and IP-routed networks. The traffic through this network may include data types such as email, file sharing or web traffic. Other forms of traffic include voice and video. These are considered as sensitive forms of traffic. They often require guaranteed or regulated service. In this paper, we deal in detail with two factors affecting QoS: Congestion and Queuing. Identifying and rectifying the above problems to reduce packet loss, latency and jitter on the network is the errand for the day. Few of the Queuing Algorithms like First in First out (FIFO), Priority Queuing (PQ), Round Robin and Weighted Round Robin (WRR) are explained in brief pictorially.
\end{abstract}

Keywords: Frame Relay, ATM, Ethernet and 802.1 networks, SONETS and IP-routed networks, Congestion and Queuing, First in First out (FIFO), Priority Queuing (PQ), Round Robin and Weighted Round Robin (WRR).

\section{INTRODUCTION}

Quality of Service (QoS) of a network [1] is a vital parameter which measures the ability of the network to provide improved service to network traffic over Frame Relay, ATM, Ethernet and 802.1 Networks, SONETS and IP-routed networks.[2]

Tools which have been developed to enforce QoS are

- Classification

Classification is based on identifying traffic based on service requirements. The traffic is then marked so that traffic can be differentiated.

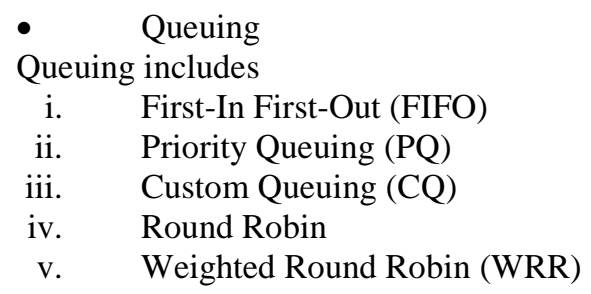

\section{Classification AND Marking}

Classification is the process of identifying and categorizing traffic into classes, typically based upon

- Incoming Interface.

- $\quad$ IP Precedence.

- $\quad$ DSCP.

- $\quad$ Source or Destination Address.

- Application.

Classification is the most fundamental QoS building block. Without classification all packets are treated the same.

Marking is carried out after classification and "colours" a packet (frame) so as to be identified and distinguished from other packets. Figure 1 shows classification and marking in the LAN with IEEE 802.1 Q. Figure 2 shows classification and marking in an Enterprise.

\section{CONGESTION AND QUEUING}

Congestion can occur in the network where there are points of speed mismatch or aggregation. Figure 3 shows Packets entering router and being forwarded by it. Network Congestion has been difficult to define quantitatively across the industry. For subscribers, it means choppy VoIP Communications, poor web browsing and frustrating online gaming performance. This is a major business threat for the Communication System Providers (CSPs). Speed mismatch 
International Journal of Advanced Research in Computer and Communication Engineering

Vol. 7, Issue 8, August 2018

are the most typical cause of congestion. Figure 4 shows the condition of Speed mismatch possibly persistent when going from LAN to WAN. Figure 5 shows Aggregation, which is another reason for Congestion. Aggregation [3] is used to provide fast and transparent recovery in case individual links fail. But choke points are created as a result of Aggregation leading to Congestion.

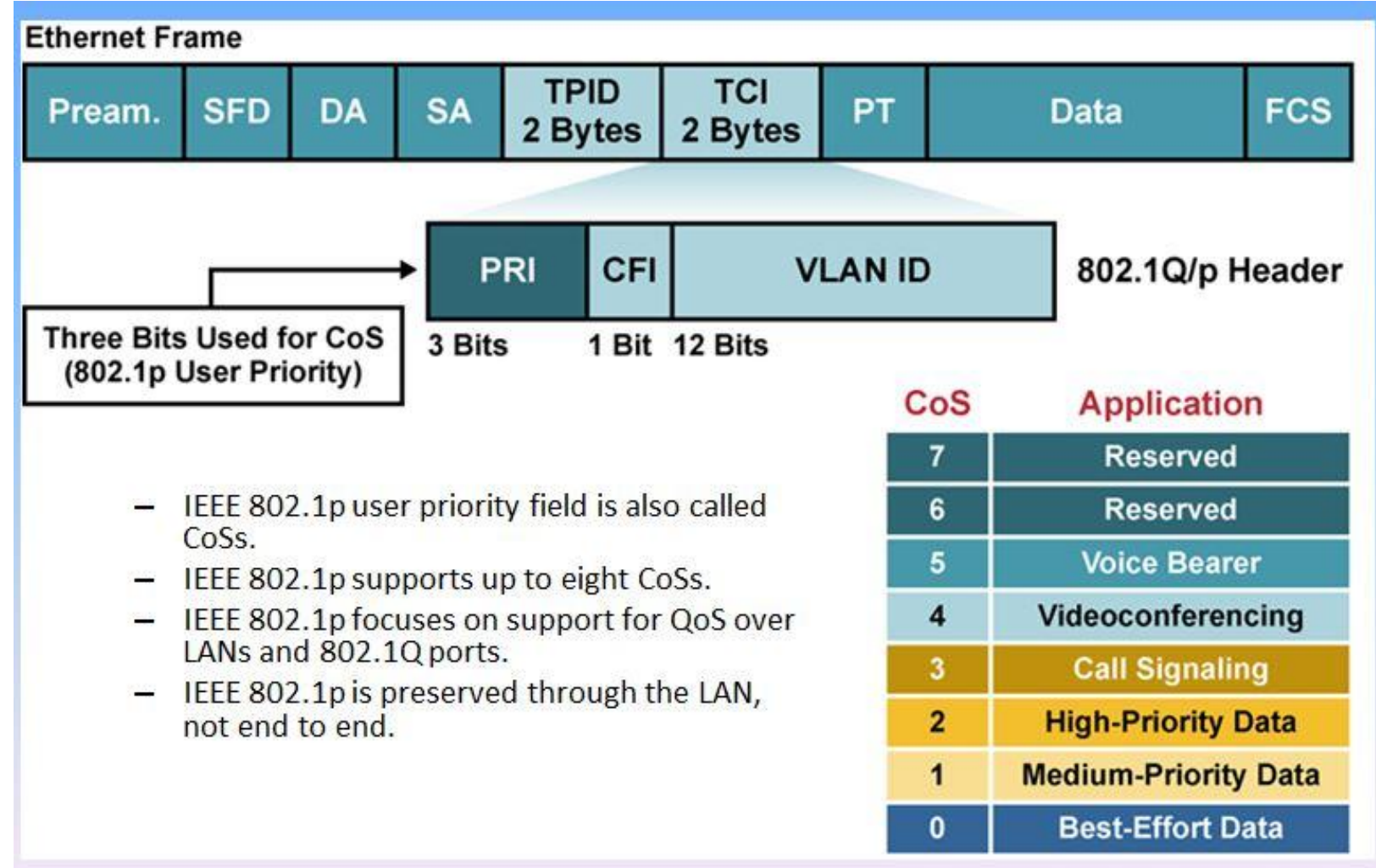

Figure 1 shows classification and marking in the LAN with IEEE 802.1 Q.

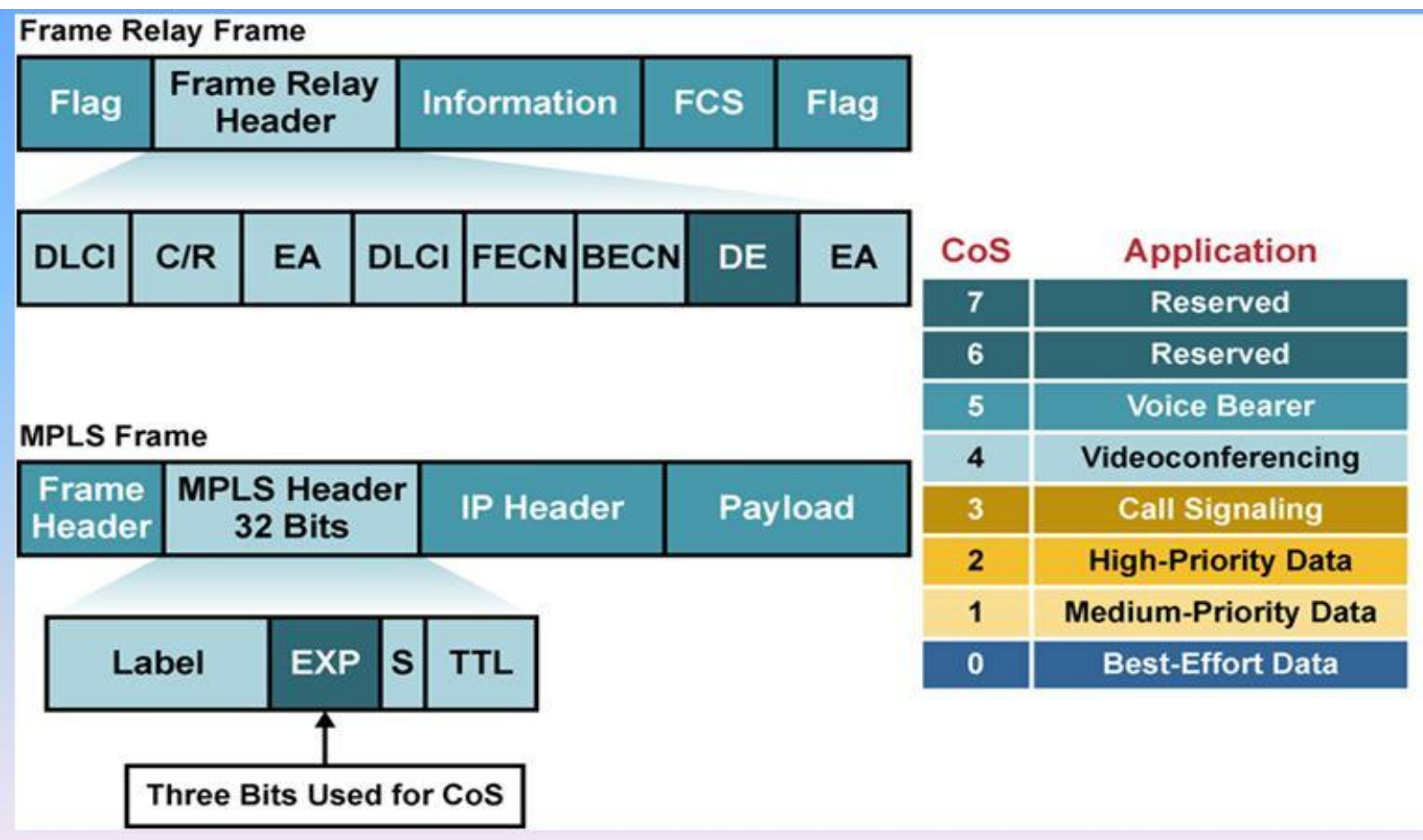

Figure 2 shows classification and marking in an Enterprise. 
International Journal of Advanced Research in Computer and Communication Engineering

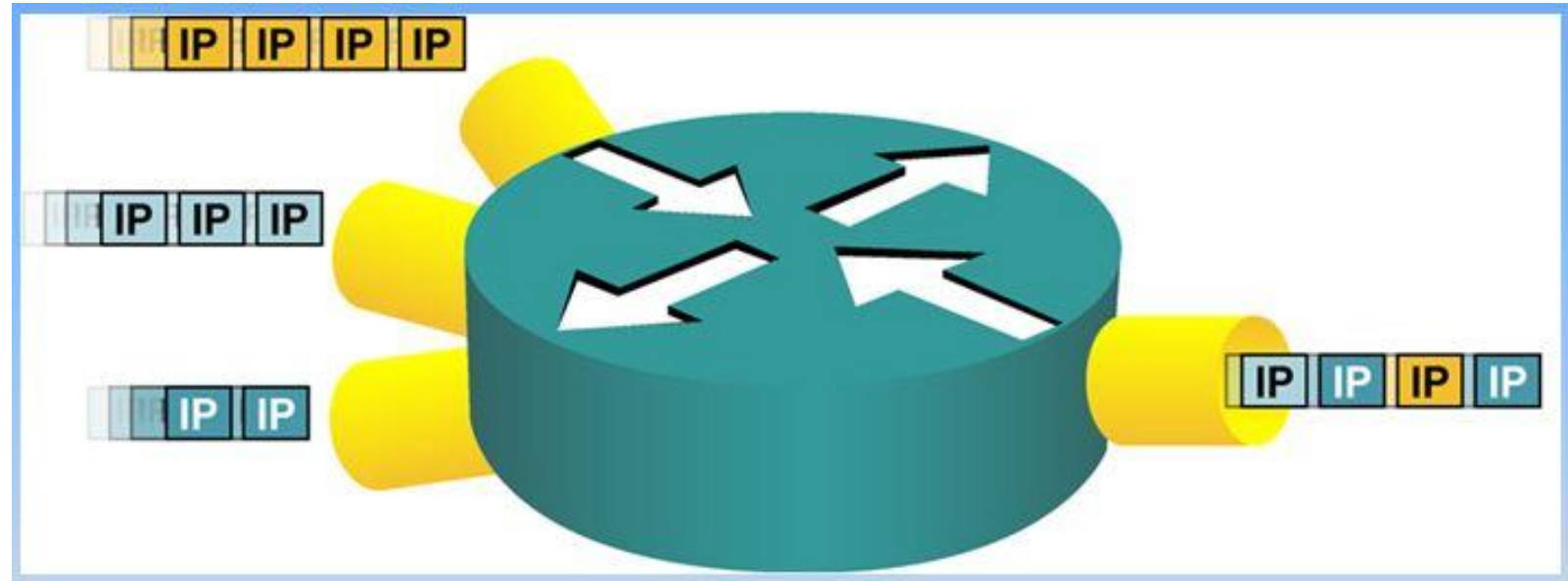

Figure 3 shows Packets entering router and being forwarded by it.

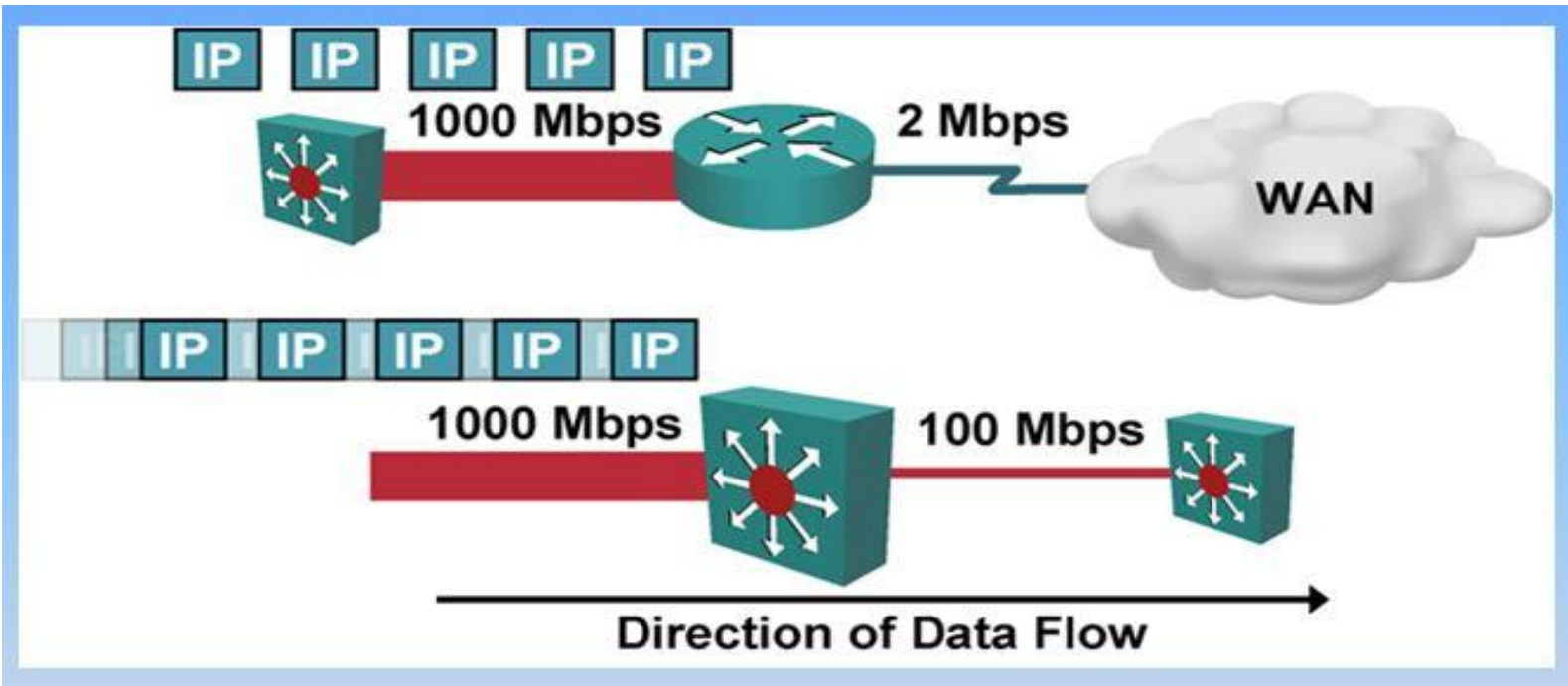

Figure 4 shows the condition of Speed mismatch possibly persistent when going from LAN to WAN.

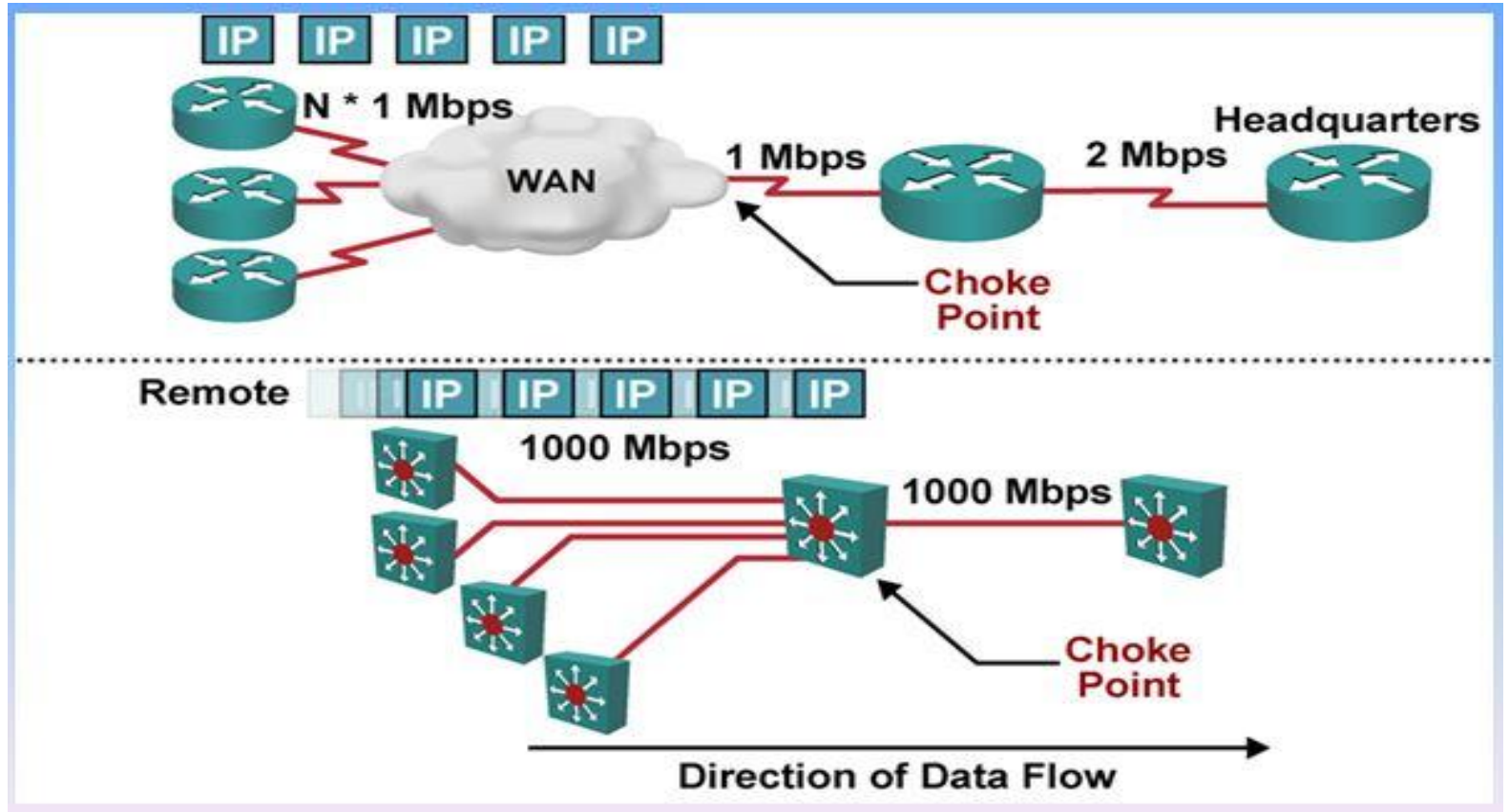

Figure 5 shows aggregation 


\section{QUEUING}

Queuing is designed to accommodate temporary congestion on an interface of a network device by storing excess packets in buffers until the bandwidth becomes available. Queuing is a Congestion-management mechanism that controls Congestion on interfaces.

The Queuing Algorithms used are:

\section{- $\quad$ First-In First-Out (FIFO)}

In this mechanism, the first packet coming in will be the first packet going out. It is the simplest of all algorithms. It consists of only one queue. All individual queues are FIFO. Figure 6 shows FIFO and packets flowing in one direction. - $\quad$ Priority Queuing $(P Q)$

Unlike FIFO, Priority Queuing uses multiple queues. It allows prioritization. Always the first queue is emptied before going to the next queue. Figure 7 shows PQ process.

Steps involved

i. $\quad$ Empty Queue No 1.

ii. If queue No 1 is empty, then dispatch one packet from queue No 2.

iii. If both queue No 1 and queue No 2 are empty then dispatch one packet from queue No 3 .

\section{- $\quad$ Round Robin and Weighted Round Robin}

Round Robin uses multiple queues. There is no prioritization. Dispatches one packet from each queue in each round. Weighted Round Robin (WRR) uses prioritization and a certain weight is assigned to each queue. Dispatch packets from each queue proportionately to an assigned weight as shown in figure 8 .
i. Dispatch up to four from queue 1.
ii. Dispatch up to two from queue 2.
iii. Dispatch one from queue 3.
iv. Go back to queue number 1 .

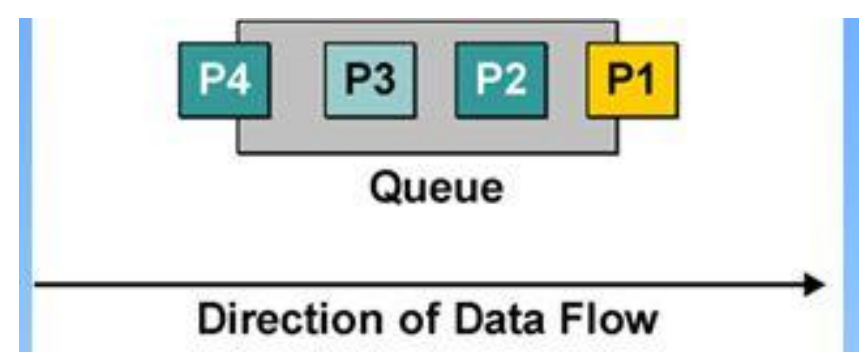

Figure 6 shows FIFO and packets flowing in one direction.

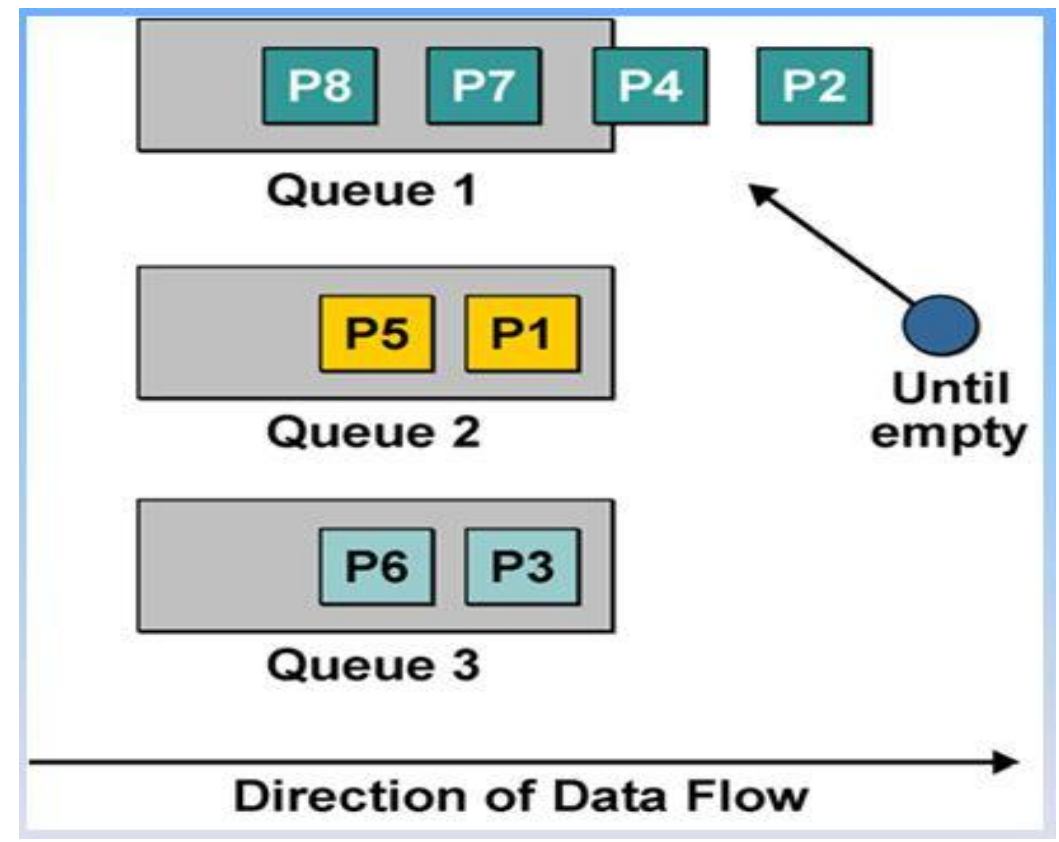

Figure 7 shows PQ process. 
Vol. 7, Issue 8, August 2018

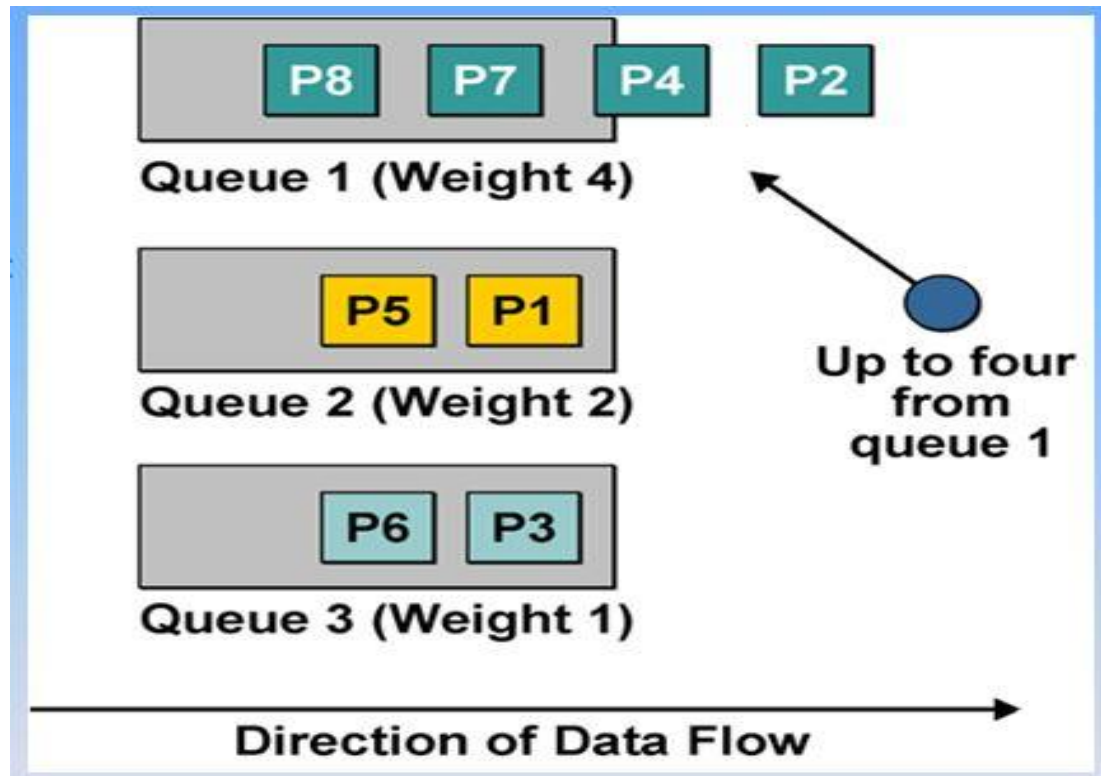

Figure 8 shows mechanism of Weighted Round Robin.

\section{CONCLUSIONS}

Delivering Quality of Service to network traffic is of great importance for critical applications and sensitive traffic like video and voice. Congestion affects QoS due to speed mismatch and aggregation. Queueing is designed to accommodate temporary congestion on an interface of a network device. Various queueing algorithms are designed to solve the problem of congestion, storing extra packets in buffers for proper bandwidth accomodation. These algorithms are explained pictorially.

\section{REFERENCES}

[1] Quality of Service Overview - Cisco- https://www.cisco.com/c/en/us/td/docs/ios/12_2/qos/.../guide/fqos.../qcfintro.pdf

[2] Layer 2 Local Switching - Cisco- https://www.cisco.com/c/en/us/td/docs/ios-xml/ios/wan.../wan-12-lcl-swng.pdf

[3] Individual QoS versus Aggregate QoS - IEEE Computer Society-

https://www.computer.org/csdl/trans/nt/2005/02/01424045.pdf

[4] Efficient fair queuing algorithms for packet-switched networks-IEEE- DOI-10.1109/90.664266

\section{BIOGRAPHY}

VISHESH S born on $13^{\text {th }}$ June 1992, hails from Bangalore (Karnataka) and has completed B.E in Telecommunication Engineering from VTU, Belgaum, Karnataka in 2015. He also worked as an intern under Dr. Shivananju BN, former

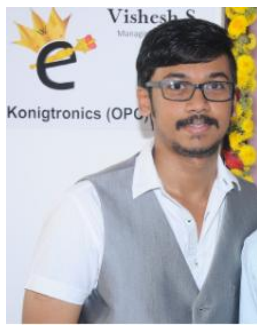

Research Scholar, Department of Instrumentation, IISc, Bangalore. His research interests include Embedded Systems, Wireless Communication, BAN and Medical Electronics. He is also the Founder and Managing Director of the corporate company Konigtronics Private Limited. He has guided over a hundred students/interns/professionals in their research work and projects. He is also the co-author of many International Research Papers. He is currently pursuing his MBA in eBusiness and PG Diploma in International Business. Presently Konigtronics Private Limited has extended its services in the field of Software Engineering and Webpage Designing. Konigtronics also conducts technical and non-technical workshops on various topics. 